\title{
A Mean Square Stability Test for Markovian Jump Linear Systems
}

C. NESPOLI ${ }^{1}$, Departamento de Matemática, Estatística e Computação, FCT/UNESP, 19010-060 Presidente Prudente, SP, Brasil

J.B.R. Do VAL 2 , Departamento de Telemática, Faculdade de Engenharia Elétrica, UNICAMP, 13081-970 Campinas, SP, Brasil

\begin{abstract}
This paper proposes a test for the mean square stability problem for discrete-time linear systems subject to random jumps in the parameters, described by an underlying finite-state Markov chain. In the model studied, the horizon of the problem is given by a stopping time $\tau_{\Delta}$, associated with the occurrence of a crucial failure after which the system is brought to a halt for maintenance. The usual stochastic stability concepts and associated results are not indicated, since they are tailored to purely infinite horizon problems. Using the concept named stochastic $\tau$-stability, equivalent conditions to ensure the stochastic stability of the system until the occurrence of $\tau_{\Delta}$ is obtained. These conditions lead to a test that benefits from the chain structure for proposing a simpler decomposition algorithm for the mean square stability verification for infinite horizon problems.
\end{abstract}

Keywords. Discrete-time Markov Jump Linear Systems; Mean Square Stability; Stopping Times.

\section{Introduction}

Consider a discrete-time linear system subject to abrupt parameter changes that can be modelled via a discrete-time finite-state Markov chain. Here, these changes are associated with failures or repairs of the running system. This class of systems is known in the literature as discrete-time Markovian jump linear systems (MJLS); see (2.1) and (2.2) (see, for instance, [2] and references therein).

The study of MJLS, and in particular, the study of stochastic stability of MJLS has continuously attracted the attention of many researchers. Ji et al [6] established conditions to test second order stability through a set of coupled algebraic matrix equations, known as coupled Lyapunov equations. Costa and Fragoso [3], using Kronecker product, obtained necessary and sufficient conditions for mean square stability of these systems with additive noise included. However, these studies are concerning to infinite time horizon problems. A situation of interest arises when one studies this class of systems until the occurrence of a stopping time $\tau$ of the

\footnotetext{
${ }^{1}$ cnespoli@fct.unesp.br.

2jbosco@dt.fee.unicamp.br.
} 
joint process $\left\{x_{k}, \theta_{k}, k \geq 0\right\}$ modelled by (2.1) and (2.2). The stopping time $\tau$ may represent interesting situations from the point of view of applications. The stochastic stability analysis for the case where $\tau$ represents the occurrence of a fixed number $N$ of failures or repairs of the system $\left(\tau=T_{N}\right)$ has been developed in [4]. In that work, it was introduced a new concept called stochastic $\tau$-stability (see Definition 2.1), tailored for problems in which the horizon of the problem coincides with the occurrence of a stopping time. In this paper we consider particularly the case where $\tau=\tau_{\Delta}$ indicates the occurrence of a "crucial failure", which may occur after a random number of failures, and after which the system is brought to a halt for maintenance. This fatal occurrence is represented by a jump into to the absorbent state $\Delta$ in an augmented Markov chain. Necessary and sufficient conditions to ensure the stochastic $\tau$-stability were provided in this case, which differ from those presented in [6] and [3] of comparative interest. These conditions yield a simpler verification test for mean square stability of MJLS with infinite time horizon.

\section{Notation and Problem Formulation}

Consider the discrete-time homogeneous Markov chain $\left\{\theta_{k} ; k \geq 0\right\}$ with space state $\mathfrak{X}=\{1, \ldots, s\} \cup\{\Delta\}\left(\Delta\right.$ is a absorbent state), initial distribution $\mu=\left(\mu_{1}, \ldots, \mu_{i}\right)$ where $\mu_{i}=P\left(\theta_{0}=i\right)$, for all $i \in \mathfrak{X}$ and transition probability matrix $\mathbb{P}=\left[p_{i j}\right]$ where

$$
p_{i j}:=P\left(\theta_{k+1}=j \mid \theta_{k}=i\right), \forall i, j \in \mathfrak{X}, k=0,1, \ldots
$$

Throughout this paper the following notation is adopted. $\mathbb{R}^{n}$ denotes the $n$ dimensional real space and $\mathcal{M}^{m \times n}\left(\mathcal{M}^{m}\right)$ and the normed linear space of all $m \times n$ $(m \times m)$ real matrices. The transpose of matrix $U$ is indicated by $U^{\prime}$ and a positive semidefinite matrix (positive definite) is represented by $U \geq 0(U>0)$. Thus, the closed (opened) convex cone of all the positive semidefinite (positive definite) matrices in $\mathcal{M}^{m}$ is denoted by $\mathcal{M}^{m 0}=\left\{U \in \mathcal{M}^{m}: U=U^{\prime} \geq 0\right\}\left(\mathcal{M}^{m+}\right)$. The linear space of all sequences of $r$ real matrices in $\mathcal{M}^{m \times n}\left(\overline{\mathcal{M}}^{m}\right)$ is represented by $\mathbb{M}^{m \times n}=\left\{\mathbf{U}=\left(U_{1}, \cdots, U_{r}\right): U_{i} \in \mathcal{M}^{m \times n}, i \in \mathfrak{X}\right\}\left(\mathbb{M}^{m}\right)$. For the sake of notational simplification, $\mathbb{M}^{m 0}$ is written when $U_{i} \in \mathcal{M}^{m 0}$, for all $i \in \mathfrak{X}$ and $\mathbb{M}^{m+}$ is written when $U_{i} \in \mathcal{M}^{m+}$. The standard vector norm in $\mathbb{R}^{n}$ is indicated by $\|\cdot\|$ and the corresponding induced norm of matrix $U$ by $\|U\|$. In addition, $r_{\sigma}(U)$ indicates the spectral radius of $U \in \mathcal{M}^{m}$, and $a \wedge b$ denotes $\min \{a, b\}$. Let $\mathbb{1}_{\{.\}}$be the Dirac measure. For $\mathbf{U} \in \mathbb{M}^{m 0}$, the following operator is defined

$$
\mathcal{E}_{i}^{\Delta}(\mathbf{U})=\sum_{j \neq i, j \neq \Delta} p_{i j} U_{j}
$$

Writing $H=\left[h_{1} \ldots h_{n}\right] \in \mathcal{M}^{m \times n}, \mathbf{H}=\left(H_{1}, H_{2}, \ldots, H_{r}\right) \in \mathbb{M}^{m \times n}$, define

$$
\varphi(H):=\left[\begin{array}{c}
h_{1} \\
\vdots \\
h_{n}
\end{array}\right] \in \mathbb{R}^{m n}, \hat{\varphi}(\mathbf{H}):=\left[\begin{array}{c}
\varphi\left(H_{1}\right) \\
\vdots \\
\varphi\left(H_{r}\right)
\end{array}\right] \in \mathbb{R}^{(r m n)} .
$$


For $g_{i} \in \mathbb{R}^{n^{2}}, i \in\{1, \ldots, r\}$, consider $\hat{\varphi}_{j}^{-1}\left(\left[\begin{array}{c}g_{1} \\ \vdots \\ g_{r}\end{array}\right]\right):=\varphi^{-1}\left(g_{j}\right), \quad j \in\{1, \ldots, r\}$.

Let the discrete-time Markovian Jump Linear Systems (MJLS) defined on the fundamental probability space $\left(\Omega, \mathfrak{F},\left\{\mathfrak{F}_{k}\right\}, P\right)$,

$$
\mathcal{S}: \quad x_{k+1}=A_{\theta_{k}} x_{k}, \quad x_{0} \in \mathbb{R}^{n}, \theta_{0} \sim \mu
$$

where $\left\{x_{k}, \theta_{k} ; k \geq 0\right\}$ is the process state taking values in $\mathbb{R}^{n} \times \mathfrak{X}$. When $\theta_{k}=i$, the MJLS evolves according to the "ith mode", namely, $A_{\theta_{k}}=A_{i} \in \mathcal{M}^{n}$.

In this work we study the stability for MJLS as in (2.2) until the occurrence of a crucial failure event after which the system is brought to a halt for maintenance. This crucial failure is associated with the time of the jump into the absorbent state $\Delta$ represented by the stopping time $\tau_{\Delta}$. Initially, a mixed and intermediary case defined by the minimum between the occurrence of a finite number $N$ of failures or repairs $T_{N}$ and the aforementioned stopping time $\tau_{\Delta}$ is also studied. Despite of being interesting in itself, it is also used here as a strategy for studying the case $\tau_{\Delta}$. In this scenario, since the occurrence of any fault or repair is associated with the jump of the Markov chain state, define the sequence $\mathcal{T}^{N}=\left\{T_{n} ; n=0,1, \ldots, N\right\}$ of $\left\{\mathfrak{F}_{k}\right\}$-stopping times

$$
\begin{aligned}
& T_{0}=0 \\
& T_{n}=\min \left\{k>T_{n-1}: \theta_{k} \neq \theta_{T_{n-1}}\right\}, n=1,2, \ldots, N .
\end{aligned}
$$

For all $m \geq 1$ and $i \in \mathfrak{X}$, it is easy to verify that

$$
P\left(T_{1}=m \mid \theta_{0}=i\right)=p_{i i}{ }^{m-1}\left(1-p_{i i}\right) \mathbb{1}_{\left\{p_{i i}<1\right\}} \text {. }
$$

Note that $P\left(T_{1}=1 \mid \theta_{0}=i\right)=1$ and $P\left(T_{1}=+\infty \mid \theta_{0}=i\right)=1$, whenever $p_{i i}=0$ and $p_{i i}=1$, respectively. From this perspective is adopted the stochastic $\tau$-stability concept introduced in [4] that is tailored to the announced problems.

Definition 2.1. Consider a stopping time $\tau$ with respect to $\left\{\mathfrak{F}_{k}\right\}$. Then, the MJLS $\mathcal{S}$ is

i) Stochastically $\tau$-stable $(\tau-S S)$ if for each initial condition $x_{0}$ and initial distribution $\mu$

$$
E\left[\sum_{k \geq 0}\left\|x_{k}\right\|^{2} \mathbb{1}_{\{\tau \geq k\}}\right]<\infty
$$

ii) $\mathbf{M}$ ean $\mathbf{S}$ quare $\tau$-Stable ( $\tau$-MSS) if for each initial condition $x_{0}$ and initial distribution $\mu$

$$
E\left[\left\|x_{k}\right\|^{2} \mathbb{1}_{\{\tau \geq k\}}\right] \rightarrow 0 \quad \text { when } k \rightarrow \infty
$$

The result below was proved in [4] and will be used latter. 
Proposition 1. Let $\tau \in \mathcal{T}^{N}$. The following assertions are equivalent:

i) The MJLS $\mathcal{S}$ is $\tau$-SS.

ii) The MJLS $\mathcal{S}$ is $\tau$-MSS.

iii) For any given set of matrices $\mathbf{Q} \in \mathbb{M}^{n+}$, there exists a unique set of matrices $\mathbf{L} \in \mathbb{M}^{n+}$, satisfying the Lyapunov equations

$$
p_{i i} A_{i}^{\prime} L_{i} A_{i}-L_{i}+Q_{i}=0, \quad \forall i \in \mathfrak{X} .
$$

iv) $r_{\sigma}\left(p_{i i}^{1 / 2} A_{i}\right)<1, \quad \forall i \in \mathfrak{X}$.

\section{Preliminary Results}

Consider the stopping time $\tau_{\Delta}$, defined as the hitting-time of $\Delta$, i. e., the time of first visit to state $\Delta$,

$$
\tau_{\Delta}=\inf \left\{n \geq 1: \theta_{n}=\Delta\right\} .
$$

Note that $\left\{T_{1}=k, \tau_{\Delta} \leq T_{1}\right\}$ and $\left\{T_{1}=k, \tau_{\Delta}>T_{1}\right\}$ are equivalent to $\left\{\theta_{k}=\Delta, \theta_{k} \neq\right.$ $\left.\theta_{k-1}=\cdots=\theta_{1}=\theta_{0}\right\}$ and $\left\{\theta_{k} \neq \Delta, \theta_{k} \neq \theta_{k-1}=\cdots=\theta_{1}=\theta_{0}\right\}$, respectively. Thus, for all $k \geq 1$ and $i, j \in \mathfrak{X}$ the probabilities bellow follows immediately

$$
\begin{gathered}
P\left(T_{1}=k, \tau_{\Delta} \leq T_{1} \mid \theta_{0}=i\right)=p_{i i}^{k-1} p_{i \Delta} \mathbb{1}_{\{i \neq \Delta\}}, \\
P\left(\theta_{k}=j, T_{1}=k, \tau_{\Delta}>T_{1} \mid \theta_{0}=i\right)=p_{i i}^{k-1} p_{i j} \mathbb{1}_{\{j \neq i \neq \Delta\}} .
\end{gathered}
$$

In the sequence, define the functional

$$
V^{n}\left(x_{0}, \theta_{0}\right)=: E\left[\sum_{k=0}^{\tau-1}\left\|x_{k}\right\|^{2}+x_{\tau}^{\prime} S_{\theta_{\tau}} x_{\tau} \mid x_{0}, \theta_{0}\right], \quad n=0, \ldots, N,
$$

by setting $V^{0}(x, \theta)=: x^{\prime} S_{\theta} x$ and $V^{n}(x, \Delta):=x^{\prime} S_{\Delta} x$ for all $n$. The next lemma, which the proof idea is presented in appendix, will be useful.

Lemma 3.1. Let $\tau=\tau_{\Delta} \wedge T_{N}$. If $\mathcal{S}$ is $\tau$-SS, then (3.3) can be expressed as

$$
V^{n}\left(x_{0}, \theta_{0}\right)=x_{0}^{\prime} S_{\theta_{0}}^{n} x_{0}, \text { with } n=1, \ldots, N,
$$

where the matrices $S_{i}^{n} \in \mathbb{M}^{n+}$ are obtained recursively as

$$
S_{i}^{n}-p_{i i} A_{i}^{\prime} S_{i}^{n} A_{i}=I_{n}+A_{i}^{\prime} p_{i \Delta} S_{\Delta} A_{i}+A_{i}^{\prime} \mathcal{E}_{i}^{\Delta}\left(\mathbf{S}^{\mathbf{n}-\mathbf{1}}\right) A_{i},
$$

with $\mathbf{S}^{\mathbf{0}}=\mathbf{S}$

Considering that $\{\tau \geq k\}=\left\{\tau_{\Delta} \wedge T_{N} \geq k\right\} \subset\left\{T_{N} \geq k\right\}$, one concludes, by using the Dominated Convergence Theorem, that the $\tau$-stability concept and the equivalences presented in Proposition 1 are applied to the mixed case $\tau=\tau_{\Delta} \wedge T_{n}$.

The result bellow follows directly from Proposition 1 and provides necessary and sufficient conditions to ensure the stochastic $\tau$-stability in the case $\tau=\tau_{\Delta} \wedge T_{n}$, $n \leq N$. 
Corollary 3.0. Let $\tau=\tau_{\Delta} \wedge T_{n}, n \leq N$. The necessary and sufficient conditions for stochastic $\tau$-stability are given by Proposition 1.

Proof. The sufficiency follows immediately by the fact that $\tau \leq T_{N}$. In this sense, it is enough to observe that $\tau_{\Delta}$ coincides with some of the jump times $T_{n}$, and also the minimum $\tau=\tau_{\Delta} \wedge T_{n}, n \leq N$ coincides with $T_{n}$ for some $n \leq N$. The necessity is based on Lemma 3.1. Setting $S \equiv I_{n}$ in functional (3.3) we conclude that

$$
V^{n}\left(x_{0}, \theta_{0}\right)=E\left[\sum_{k \geq 0}\left\|x_{k}\right\|^{2} \mathbb{1}_{\{\tau \geq k\}} \mid x_{0}, \theta_{0}\right]
$$

and thus

$$
S_{i}^{n}-p_{i i} A_{i}^{\prime} S_{i}^{n} A_{i}>0
$$

holds for each $n \leq N$. Hence, the result relies on the Lyapunov Stability theory.

\section{Conditions for $\tau_{\Delta}$-stability}

In this section are presented the necessary and sufficient conditions for $\tau$-stability when $\tau=\tau_{\Delta}$. These conditions are obtained by setting $\tau=\lim _{N \rightarrow \infty}\left\{\tau_{\Delta} \wedge T_{N}\right\}$ in the mixed case and relying on the Dominated Convergence Theorem. In the sequel $\operatorname{Diag}\left(U_{i}\right)$, for all $U_{i} \in \mathcal{M}^{m}(i \neq \Delta)$, indicates the matrices with $U_{i}$ on the main diagonal and zero otherwise. Initially, consider the transition probability matrix $P$, block-partitioned in the form

$$
P=\left[\begin{array}{cc}
1 & 0 \\
\mathcal{B} & \mathcal{Q}
\end{array}\right] \text { where } \mathcal{B}:=\left[\begin{array}{c}
p_{1 \Delta} \\
p_{2 \Delta} \\
\vdots \\
p_{s \Delta}
\end{array}\right] \text { and } \mathcal{Q}:=\left[\begin{array}{ccc}
p_{11} & \cdots & p_{1 s} \\
p_{21} & \cdots & p_{2 s} \\
\vdots & \vdots & \vdots \\
p_{s 1} & \cdots & p_{s s}
\end{array}\right]
$$

In addition, write

$$
\begin{aligned}
\mathcal{A}_{1} & :=\operatorname{Diag}\left(A_{i} \otimes A_{i}\right)^{\prime} \operatorname{Diag}\left(\mathcal{Q} \otimes I_{n^{2}}\right), \\
\mathcal{A}_{2} & :=\operatorname{Diag}\left(A_{i} \otimes A_{i}\right)^{\prime}\left(\tilde{\mathcal{Q}} \otimes I_{n^{2}}\right), \quad \text { and } \\
\mathcal{A} & :=\mathcal{A}_{1}+\mathcal{A}_{2}=\operatorname{Diag}\left(A_{i} \otimes A_{i}\right)^{\prime}\left(\mathcal{Q} \otimes I_{n^{2}}\right),
\end{aligned}
$$

where $\tilde{Q}$ is obtained by setting the main diagonal of the matrix $\mathcal{Q}$ to zero. For any $L, K, H \in \mathcal{M}^{n}$, the next properties are valid:

$$
\begin{gathered}
(L \otimes K)^{\prime}=L^{\prime} \otimes K^{\prime}, \\
(L \otimes K)(H \otimes R)=L H \otimes K R, \\
\varphi(L K H)=\left(H^{\prime} \otimes L\right) \varphi(K) .
\end{gathered}
$$

Theorem 4.1. For $\tau=\tau_{\Delta}$ the following conditions are equivalent:

i) The $M J L S \mathcal{S}$ is $\tau$-SS.

ii) $r_{\sigma}(\mathcal{A})<1$. 
iii) $r_{\sigma}\left(\mathcal{A}_{1}\right)<1$ and $r_{\sigma}\left(\left[I_{s r^{2}}-\mathcal{A}_{1}\right]^{-1} \mathcal{A}_{2}\right)<1$.

iv) For any given set of matrices $\mathbf{Q} \in \mathbb{M}^{n+}$, there exists a unique set of matrices $\mathbf{L} \in \mathbb{M}^{n+}$, satisfying the Lyapunov equations

$$
\sum_{j=1}^{s} p_{i j} A_{i}^{\prime} L_{j} A_{i}-L_{i}+Q_{i}=0, i=1, \ldots, s
$$

or equivalently

$$
L_{i}=\hat{\varphi}_{i}^{-1}\left(\left[I_{s r^{2}}-\mathcal{A}\right]^{-1} \hat{\varphi}(\mathbf{Q})\right)
$$

Proof. Firstly, consider the relations

$$
E_{0}\left[\sum_{k \geq 0}\left\|x_{k}\right\|^{2} \mathbb{1}_{\{k \leq \tau \Delta\}}\right]=\lim _{N \rightarrow \infty} E_{0}\left[\sum_{k \geq 0}\left\|x_{k}\right\|^{2} \mathbb{1}_{\left\{k \leq \tau_{\Delta} \wedge T_{N}\right\}}\right]=\lim _{N \rightarrow \infty} x_{0} S_{\theta_{0}}^{N} x_{0}
$$

for each $\left(x_{0}, \theta_{0}\right)$, where the former follows from Dominated Convergence Theorem and the latter is valid for $\mathbf{S}^{\mathbf{N}}$ in (3.4) with $\mathbf{S}^{\mathbf{0}}=\mathbf{I}_{\mathbf{n}}$, cf. Lemma 3.1. Using the operator $\varphi$, the expression (3.4) can be rewritten as

$$
\varphi\left(S_{i}^{n}\right)-\varphi\left(p_{i i} A_{i}^{\prime} S_{i}^{n} A_{i}\right)=\varphi\left(\hat{Q}_{i}\right)+\varphi\left(A_{i}^{\prime}{ }_{i} \mathcal{E}_{i}^{\Delta}\left(\mathbf{S}^{\mathbf{n}-\mathbf{1}}\right) A_{i}\right)
$$

where $\hat{Q}_{i}=I_{n}+A_{i}^{\prime} p_{i \Delta} S_{\Delta} A_{i}$. Using the properties (4.1) and (4.3),

$$
\left[I_{n^{2}}-p_{i i} I_{n^{2}}\left(A_{i}^{\prime} \otimes A_{i}^{\prime}\right)\right] \varphi\left(S_{i}^{n}\right)=\varphi\left(\hat{Q}_{i}\right)+\sum_{j \neq i, j \neq \Delta} p_{i j} I_{n^{2}}\left(A_{i}^{\prime} \otimes A_{i}^{\prime}\right) \varphi\left(S_{j}^{n-1}\right)
$$

for all $i=1, \ldots, s$. Introducing the matrices $Q, \tilde{Q}$ and the operator $\hat{\varphi}$, the equation (4.7) becomes equivalent to

$$
\left[I_{s r^{2}}-\mathcal{A}_{1}\right] \hat{\varphi}\left(\mathbf{S}^{n}\right)=\hat{\varphi}(\hat{\mathbf{Q}})+\mathcal{A}_{2} \hat{\varphi}\left(\mathbf{S}^{n-1}\right) .
$$

(iii) $\Rightarrow$ (i) A necessary condition for the convergence of (4.8) is the non-singularity of $I_{s r^{2}}-\mathcal{A}_{1}$, and that $r_{\sigma}\left(\left[I_{s r^{2}}-\mathcal{A}_{1}\right]^{-1} \mathcal{A}_{2}\right)<1$, see [8, theo 10.1.1]. Thus, (iii) implies the convergence of the recursive equation (4.8), and the $\tau$-stability follows from (4.6).

(i) $\Rightarrow$ (iv) By hypothesis and (4.6), there exists $\mathbf{L} \in \mathbb{M}^{n+}$ such that

$$
E\left[\sum_{k \geq 0}\left\|x_{k}\right\|^{2} \mathbb{1}_{\{k \leq \tau\}}\right]=x_{0} L_{\theta_{0}} x_{0}
$$

and $\mathbf{L}$ satisfies (3.4), or equivalently, (4.8):

$$
\left[I_{s r^{2}}-\mathcal{A}_{1}\right] \hat{\varphi}(\mathbf{L})=\hat{\varphi}(\hat{\mathbf{Q}})+\mathcal{A}_{2} \hat{\varphi}(\mathbf{L}) .
$$

According to definition of $\mathcal{A}$, the preceding equation can be rewritten as

$$
\left[I_{s r^{2}}-\mathcal{A}\right] \hat{\varphi}(\mathbf{L})=\hat{\varphi}(\hat{\mathbf{Q}}),
$$

which provides (4.4) by inspection. The uniqueness of $\mathbf{L}$ implies that $I_{s r^{2}}-\mathcal{A}$ is invertible, completing this part of the proof. 
(iv) $\Rightarrow$ (ii) Since the expressions in (iv) are equivalent to (4.10), the uniqueness of $\mathbf{L}$ implies that $\operatorname{Eig}(\mathcal{A}) \neq 1$. The fact that $r_{\sigma}(\mathcal{A})<1$ is originated from the property that $\mathbf{L} \in \mathbb{M}^{n+}$ for all $\mathbf{Q} \in \mathbb{M}^{n+}$ (see [5, pg. 538]).

(ii) $\Rightarrow$ (iii) If (ii) is verified, then (4.5) defines $\mathbf{L} \in \mathbb{M}^{n+}$ uniquely. Since (4.4) and (4.5) are equivalent, for all $\tilde{\mathbf{Q}} \in \mathbb{M}^{n+}$,

$$
p_{i i} A_{i}^{\prime} R_{i} A_{i}-R_{i}+\tilde{Q}_{i}=0 \text {, or }\left[I_{s n^{2}}-\operatorname{Diag}\left(A_{i} \otimes A_{i}\right)^{\prime} \operatorname{Diag}\left(\mathcal{Q} \otimes I_{n^{2}}\right)\right] \hat{\varphi}(\mathbf{R})=\hat{\varphi}(\tilde{\mathbf{Q}})
$$

are satisfied by a unique $\mathbf{R} \in \mathbb{M}^{n+}$. The last expression implies that $r_{\sigma}\left(\mathcal{A}_{1}\right)<1$. From (4.5), one writes

$$
\begin{aligned}
\hat{\varphi}(\mathbf{L}) & =\left[I_{s r^{2}}-\mathcal{A}\right]^{-1} \hat{\varphi}(\mathbf{Q})=\left[I_{s r^{2}}-\mathcal{A}_{1}-\mathcal{A}_{2}\right]^{-1} \hat{\varphi}(\mathbf{Q}) \\
& =\left[\left(I_{s r^{2}}-\mathcal{A}_{1}\right)\left(I_{s r^{2}}-\left(I_{s r^{2}}-\mathcal{A}_{1}\right)^{-1} \mathcal{A}_{2}\right]^{-1} \hat{\varphi}(\mathbf{Q})\right. \\
& =\left[I_{s r^{2}}-\left(I_{s r^{2}}-\mathcal{A}_{1}\right)^{-1} \mathcal{A}_{2}\right]^{-1}\left(I_{s r^{2}}-\mathcal{A}_{1}\right)^{-1} \hat{\varphi}(\mathbf{Q}) .
\end{aligned}
$$

Hence $\operatorname{Eig}\left(\left(I_{s r^{2}}-\mathcal{A}_{1}\right)^{-1} \mathcal{A}_{2}\right) \neq 1$, and since that $\mathbf{L} \in \mathbb{M}^{n+}$ for all $\mathbf{Q} \in \mathbb{M}^{n+}$, the second condition about the spectral radius in (iii) results proven.

Remark 1. Note that the conditions for $\tau$-stability presented in Proposition 1 are given in terms of uncoupled Lyapunov equations. On the other hand, although the conditions for $\tau$-stability provided in Theorem 4.1 are given in terms of coupled Lyapunov equations, they differ from the criteria for purely infinite horizon of comparative interest, see [6], since that $\sum_{j}^{s} p_{i j} \leq 1$. Besides, the matrix $\mathcal{A}$ in (ii) involves only the matrix $\mathcal{Q}$. Therefore, this condition also differs from that proposed in [3] for mean square stability, namely, $r_{\sigma}\left(\left(P \otimes I_{n^{2}}\right) \operatorname{Diag}\left(A_{i} \otimes A_{i}\right)^{\prime}\right)<1$, which involves the full probability matrix. Consequently $\mathcal{A}$ present contractive properties more favorable, as we can confirm through the next example.

Example 1. Let the independent Markov chains $\left\{\alpha_{k} ; k \geq 0\right\}$ and $\left\{\gamma_{k} ; k \geq 0\right\}$ with state space $\bar{S}=\{1, \ldots, s\}$ and $S^{\prime}=\{g, f\}$, and transition matrices $\overline{\bar{P}}:=$ $\left[\bar{p}_{i j}\right]$ and $\mathbb{Q}:=\left[q_{i j}\right]$, respectively, with $q_{g g}=\rho<1$ and $q_{f f}=1$. Consider the composed chain $\left\{\theta_{k}=\left(\alpha_{k}, \gamma_{k}\right) ; k \geq 0\right\}$ with state space $S=\{(i, f),(j, g), i, j=$ $1, \ldots, s\}$, in which $\Delta=\{(i, f), i=1, \ldots, s\}$, and transition matrix $P$ where

$$
\begin{gathered}
P\left(\theta_{k+1}=(j, g) \mid \theta_{k}=(i, g)\right)=\bar{p}_{i j} \rho \\
\left.P\left(\theta_{k+1}=\Delta\right) \mid \theta_{k}=(i, g)\right)=\bar{p}_{i j}(1-\rho) \\
\left.P\left(\theta_{k+1}=\Delta\right) \mid \theta_{k}=\Delta\right)=1 .
\end{gathered}
$$

The matrix $P$ can be written as $P=\left[\begin{array}{ll}\mathbf{1} & \mathbf{0} \\ \hat{\mathcal{B}} & \hat{\mathcal{Q}}\end{array}\right]$, with

$$
\hat{\mathcal{B}}=:\left[\begin{array}{ccc}
\bar{p}_{11}(1-\rho) & \ldots & \bar{p}_{1 s}(1-\rho) \\
\vdots & \vdots & \vdots \\
\bar{p}_{s 1}(1-\rho) & \ldots & \bar{p}_{s s}(1-\rho)
\end{array}\right] \text { and } \quad \hat{\mathcal{Q}}=:\left[\begin{array}{ccc}
\bar{p}_{11} \rho & \ldots & \bar{p}_{1 s} \rho \\
\vdots & \vdots & \vdots \\
\bar{p}_{s 1} \rho & \ldots & \bar{p}_{s s} \rho
\end{array}\right]
$$

Additionally, set $A_{(i, g)}=A_{i}$ and $A_{(i, f)}=A_{\Delta}$. With the objective of investigating the MSS of the system, we must calculate

$$
E\left[\left\|x_{k}\right\|^{2} \mathbb{1}_{\{\tau \geq k\}} \mid \theta_{0}=\left(i_{0}, g\right)\right],
$$


which involves the next probability

$$
\begin{gathered}
P\left(\tau \geq k, \theta_{1}=\left(i_{1}, \cdot\right), \cdots, \theta_{k-1}=\left(i_{k-1}, \cdot\right) \mid \theta_{0}=\left(i_{0}, g\right)\right)= \\
P\left(\theta_{k-1}=\left(i_{k-1}, g\right), \cdots, \theta_{1}=\left(i_{1}, g\right) \mid \theta_{0}=\left(i_{0}, g\right)\right)= \\
\rho^{k-1} \bar{p}_{i_{0} i_{1}} \bar{p}_{i_{1} i_{2}} \cdots \bar{p}_{i_{k-2} i_{k-1}} .
\end{gathered}
$$

By using (4.11) and denoting $\bar{p}_{i_{1} i_{2}} \cdots \bar{p}_{i_{k-2} i_{k-1}}$, we obtain

$$
\begin{gathered}
E\left[\left\|x_{k}\right\|^{2} \mathbb{1}_{\{\tau \geq k\}} \mid \theta_{0}=\left(i_{0}, g\right)\right]= \\
x_{0}^{\prime}\left(\sum_{i_{1}, \cdots, i_{k-1} \neq \Delta} \rho^{k-1} c A_{i_{0}}^{\prime} A_{i_{1}}^{\prime} \cdots A_{i_{k-1}}^{\prime} I_{n} A_{i_{k-1}} \cdots A_{i_{1}} A_{i_{0}}\right) x_{0} .
\end{gathered}
$$

Now, applying (4.1), (4.2), (4.3) and the operator $\varphi$, we get that

$$
\begin{aligned}
& E\left[\left\|x_{k}\right\|^{2} \mathbb{1}_{\{\tau \geq k\}} \mid \theta_{0}=\left(i_{0}, g\right)\right] \\
& =x_{0}^{\prime} \varphi^{-1}\left(\sum_{i_{1}, \cdots, i_{k-1} \neq \Delta} \rho^{k-1} c \varphi\left(A_{i_{0}}^{\prime} A_{i_{1}}^{\prime} \cdots A_{i_{k-1}}^{\prime} I_{n} A_{i_{k-1}} \cdots A_{i_{0}}\right)\right) x_{0} \\
& =x_{0}^{\prime} \varphi^{-1}\left(\left(A_{i_{0}} \otimes A_{i_{0}}\right)^{\prime} \sum_{i_{1}, \cdots, i_{k-1} \neq \Delta} \rho^{k-1} c I_{n^{2}}\left(A_{i_{1}} \otimes A_{i_{1}}\right)^{\prime} \cdots\left(A_{i_{k-1}} \otimes A_{i_{k-1}}\right)^{\prime}\right) x_{0} \\
& =x_{0}^{\prime} \varphi^{-1}\left(F G^{k-1} D\right) x_{0},
\end{aligned}
$$

where $F \in \mathcal{M}^{n^{2} \times s n^{2}}$ is the matrix with the $i_{0}$ th submatrix block non-null, namely $, F:=\left[0: 0: \cdots:\left(A_{i_{0}} \otimes A_{i_{0}}\right)^{\prime}: \cdots: 0\right], G \in \mathcal{M}^{s n^{2} \times s n^{2}}$ and $D \in \mathcal{M}^{s n^{2} \times 1}$ are defined as

$$
G:=\left(\hat{\mathcal{Q}} \otimes I_{n^{2}}\right) \operatorname{Diag}(\mathbf{A} \otimes \mathbf{A})^{\prime} \quad \text { and } \quad D:=\left[\begin{array}{c}
\varphi\left(I_{n}\right) \\
\vdots \\
\varphi\left(I_{n}\right)
\end{array}\right]
$$

Remark 2. Notice that $G \equiv \rho\left(\mathbb{P} \otimes I_{n^{2}}\right) \operatorname{Diag}(\mathbf{A} \otimes \mathbf{A})^{\prime}$ since the matrix $\hat{\mathcal{Q}}$ coincides with $\rho \mathbb{P}$. As $\rho<1$, then $\hat{\mathcal{Q}}$ it is not a probability matrix, while $\mathbb{P}$ it is. Thus, remaining evidenced the contractive characteristic of $G$. More specifically, note that $G^{k}$ is a matrix of the type $(\rho M)^{k}$ which can converge while $M^{k}$ does not converge.

\section{Mean Square Stability Test}

Suppose we are interested in the MSS of system in the form (2.1) and (2.2), with $\mathfrak{X}=\{1,2, \ldots, L\}$. Since that the set $\mathfrak{X}$ is finite, see. [1, pg. 125-132], it can be decomposed in to form $\mathcal{T}, R_{1}, \ldots, R_{l}$, where $\mathcal{T}$ contains the transient states (starting at $j$, there exists a positive probability of never returning to $j$ ) and $R_{1}, \ldots, R_{l}$ are the disjoint sets of recurrent states (starting at $j$, the probability of returning to $j$ is one). Note that each $R_{j}$ forms a closed set, i.e., no state outside it can be reached 
from any state in it. Then, the transition matrix can be put in the form

$$
P=\left[\begin{array}{ccccc}
P_{R_{1}} & 0 & \cdots & 0 & 0 \\
0 & P_{R_{2}} & \cdots & 0 & 0 \\
\vdots & & \ddots & & \vdots \\
0 & 0 & \cdots & P_{R_{l}} & 0 \\
P_{T_{1}} & P_{T_{2}} & \cdots & P_{T_{l}} & P_{\mathcal{T}}
\end{array}\right]
$$

where, for each $j=1, \ldots, l, P_{R_{j}}$ (each one taken by itself defines Markov matrices) and $P_{\mathcal{T}}$ represent the transition probabilities within the sets $R_{j}$ and $\mathcal{T}$ respectively, and $P_{T_{j}}$ represents the transition probabilities from $\mathcal{T}$ to $R_{j}$.

Costa and Fragoso [3] presented a necessary and sufficient condition for MSS, given in terms of the spectral radius of a matrix in the form $\mathcal{G}=\left(P \otimes I_{n^{2}}\right) \operatorname{Diag}(\mathbf{A} \otimes$ A $)^{\prime}$, which involves the probability matrix $P$ associated to the system. More specifically, we must obtain $r_{\sigma}(\mathcal{G})<1$.

A necessary and sufficient condition for MSS, has been obtained in [7] in terms of a Lyapunov equation in form (4.4) involving all states of the chain. On the other hand, and comparatively, a necessary and sufficient condition for the $\tau_{\Delta}$-MSS of the system has been established in Theorem 4.1 in terms of a matrix of the type $\mathcal{G}_{\Delta}=\operatorname{Diag}(\mathbf{A} \otimes \mathbf{A})^{\prime}\left(P_{\mathcal{T}} \otimes I_{n^{2}}\right)$ or a Lyapunov equation (4.4), neither of them included the absorbent state.

From this perspective, without loss of generality, we can adopt the recurrent classes as "absorbent states" and then apply the conditions (ii) and (iv) for the MSS analysis. In detail, considering the process starting at some transient state $i \in \mathcal{T}$, we can employ the results for $\tau_{\Delta}$-stability since with non-null probability the process will be absorbed by some recurrent class. In this context, it is enough to examine simultaneously the spectral radius of a matrix of the type $\mathcal{G}_{\Delta}$, involving a matrix $P_{\mathcal{T}}$ (which it is not a probability matrix ) and the spectral radio of some matrices of the type $\mathcal{G}$, involving $P_{R_{1}}, \cdots, P_{R_{l}}$.

In other words, a test for MSS can be decomposed into two parts: one test for $\tau_{\Delta}$-MSS for the transient states and some tests for MSS involving a lesser order problem.

Conversely, considering the process starting at some $j \in R_{j}$, it is enough to apply the MSS test regarding $P_{R_{j}}$.

The same arguments can be used with respect to the Lyapunov equations.

In both criteria, (using the Matlab software) the computational time expended for the MSS test here proposed has been lesser than that expended with the MSS test of comparative interest.

The following example illustrate the statements above.

Example 2. Consider the state space $\mathfrak{X}=\{1,3\} \cup\{2,7,9\} \cup\{6\} \cup\{4,5,8,10\}$, where $\mathcal{T}=\{4,5,8,10\}$ is the set of transient states, $R_{1}=\{1,3\}$ and $R_{2}=\{2,7,9\}$ are sets of recurrent states and $R_{6}=\{6\}$ is an absorbing state. 
The following matrix is adopted

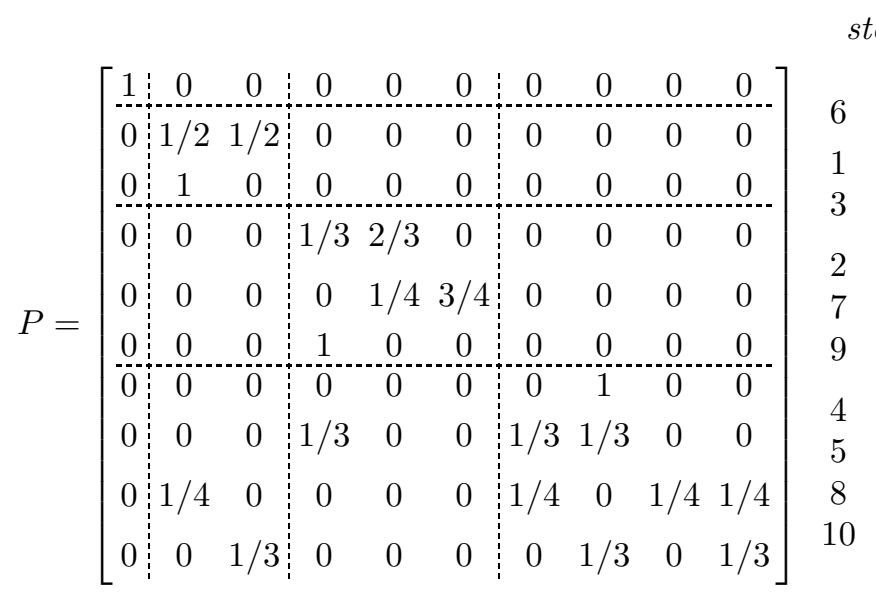

The next cases are analyzed.

Case 1: Consider $n=5$ and

$$
A_{i}=\left[\begin{array}{ccccc}
1 / 8 & 0 & 1 / 4 & 1 / 4 & 1 / 8 \\
0 & 1 / 8 & 1 / 3 & 1 / 8 & 1 / 4 \\
1 / 5 & 1 / 3 & 1 / 4 & 1 / 4 & 1 / 4 \\
1 / 3 & 1 / 3 & 1 / 3 & 1 / 8 & 1 / 4 \\
1 / 8 & 0 & 1 / 4 & 1 / 4 & 1 / 8
\end{array}\right]
$$

with $i=1, \ldots, 10$.

Case 2: Consider $n=10$ and the matrices $A_{i}, i=1, \ldots, 10$, generated by the routine rand of Matlab.

Adopting the MSS test proposed in [3], we must examine the spectral radio of the matrix $\mathcal{G}=\left(P \otimes I_{n^{2}}\right) \operatorname{Diag}(\mathbf{A} \otimes \mathbf{A})^{\prime}$, with dimension $10 n^{2} \times 10 n^{2}$, more specifically, $250 \times 250$ and $1000 \times 1000$, respectively for cases 1 and 2 . Using the routine "cputime" of Matlab, the mean execution time in these calculous was approximately 0.575 s for case 1 , with $r_{\sigma}(\mathcal{G})=0.9613$ (the system is $M S S$ ). For case 2 it was approximately $38.95 \mathrm{~s}$.

Now, adopting the $\tau$-MSS proposed here, it is enough to analyze the spectral radio of the matrix $\mathcal{G}_{\Delta}=\operatorname{Diag}(\mathbf{A} \otimes \mathbf{A})^{\prime}\left(P_{\mathcal{T}} \otimes I_{n^{2}}\right)$ with dimension $4 n^{2} \times 4 n^{2}$, and simultaneously, apply the MSS test as above for the remaining states. That test involves the probability matrices $P_{R_{1}}, P_{R_{2}}$ and $P_{R_{6}}$, and calculous of the spectral radios of the type $\mathcal{G}$ matrices with dimension $3 n^{2} \times 3 n^{2}, 2 n^{2} \times 2 n^{2}$ and $n^{2} \times n^{2}$, respectively. Regarding the mean running times they were 0.0531 s for case 1 and 4.375 s for case 2.

Resumo. Este artigo propõe um teste para estabilidade em média quadrática de sistemas li-neares com saltos nos parâmetros, descritos por uma cadeia de Markov com espaço de estados finito. No modelo estudado, um tempo de parada $\tau_{\Delta}$, associado à ocorrência de uma falha grave que leva à paralização do sistema, é adotado 
como horizonte do problema. Os conceitos de estabilidade estocástica assim como os resultados encontrados na literatura não são indicados, uma vez que referemse a problemas com horizonte infinito puro. Através do conceito denominado $\tau$ estabilidade estocástica, são obtidas condições necessárias e suficientes para garantir a estabilidade do sistema até a ocorrência de $\tau_{\Delta}$. Estas condições conduzem a um teste que se beneficia da estrutura da cadeia e permite uma decomposição para verificação de estabilidade em média quadrática para problemas com horizonte infinito, o qual induz métodos algorítmicos mais simples.

\section{A Proof of Lemma 3.1}

Proof. For simplicity, write $E_{0}[\cdot]$ instead of $E\left[\cdot \mid x_{0}, \theta_{0}\right]$. The idea consists in defining the functional

$$
\begin{gathered}
x_{0}^{\prime} S_{\theta_{0}}^{n} x_{0}=: E_{0}\left[\left(\sum_{k=0}^{T_{n}-1}\left\|x_{k}\right\|^{2}+x_{T_{n}}^{\prime} S_{\theta_{T_{n}}} x_{T_{n}}\right) \mathbb{1}_{\left\{\tau_{\Delta}>T_{n}\right\}}\right]+ \\
E_{0}\left[\left(\sum_{k=0}^{\tau_{\Delta}-1}\left\|x_{k}\right\|^{2}+x_{\tau_{\Delta}}^{\prime} S_{\theta_{\tau_{\Delta}}} x_{\tau_{\Delta}}\right) \mathbb{1}_{\left\{\tau_{\Delta} \leq T_{n}\right\}}\right]
\end{gathered}
$$

and employ an induction argument on $n$. For $n=1$,

$$
\begin{gathered}
V^{1}\left(x_{0}, \theta_{0}\right)=x_{0}^{\prime} S_{\theta_{0}}^{1} x_{0}= \\
E_{0}\left[\sum_{k=0}^{T_{1}-1}\left\|x_{k}\right\|^{2}+x_{T_{1}}^{\prime} S_{\theta_{T_{1}}} x_{T_{1}} \mathbb{1}_{\left\{\tau_{\Delta}>T_{1}\right\}}+x_{T_{1}}^{\prime} S_{\Delta} x_{T_{1}} \mathbb{1}_{\left\{\tau_{\Delta} \leq T_{1}\right\}}\right] .
\end{gathered}
$$

Applying (2.3), (3.2) and (3.1, one obtains

$$
\begin{gathered}
x_{0}^{\prime} S_{\theta_{0}}^{1} x_{0}=\sum_{k=0}^{\infty} p_{i i}^{k}\left(\left\|A_{i}^{k} x_{0}\right\|^{2}+\left\|\left(A_{i}^{\prime k+1} \mathcal{E}_{i, \Delta}(\mathbf{S}) A_{i}^{k+1}\right)^{1 / 2} x_{0}\right\|^{2}+\right. \\
\left.\left\|\left(A_{i}^{\prime k+1} p_{i \Delta} S_{\Delta} A_{i}^{k+1}\right)^{1 / 2} x_{0}\right\|^{2}\right) .
\end{gathered}
$$

Then, since $x_{0}$ is arbitrary, fixing $\rho=: I_{n}+A_{i}^{\prime} p_{i \Delta} S_{\Delta} A_{i}+A_{i}^{\prime} \mathcal{E}_{i}^{\Delta}(\mathbf{S}) A_{i}$, we get that

$$
\begin{aligned}
S_{i}^{1} & =\sum_{k=0}^{\infty} p_{i i}^{k} A_{i}^{\prime k} \rho A_{i}^{k}=\rho+\sum_{k=1}^{\infty} p_{i i}^{k} A_{i}^{\prime k} \rho A_{i}^{k} \\
& =\rho+\sum_{k=0}^{\infty} p_{i i}^{k+1} A_{i}^{\prime k+1} \rho A_{i}^{k}=\rho+p_{i i} A_{i}^{\prime}\left(\sum_{k=0}^{\infty} p_{i i}^{k} A_{i}^{\prime k} \rho A_{i}^{k}\right) A_{i} .
\end{aligned}
$$

Thus, recognizing $S_{i}^{1}$ in brackets,

$$
S_{i}^{1}-p_{i i} A_{i}^{\prime} S_{i}^{1} A_{i}=I_{n}+A_{i}^{\prime} p_{i \Delta} S_{\Delta} A_{i}+A_{i}^{\prime} \mathcal{E}_{i}^{\Delta}(\mathbf{S}) A_{i},
$$

completing the proof for $n=1$, for the case $0<p_{i i} \leq 1$. For the case $p_{i i}=0$, since $P\left(T_{1}=1 \mid \theta_{0}=i\right)=1$,

$$
x_{0}^{\prime} S_{\theta_{0}}^{1} x_{0}=\sum_{k=0}^{\infty}\left(\left\|x_{0}\right\|^{2}+\left\|\left(A_{i}^{\prime} \mathcal{E}_{i, \Delta}(\mathbf{S}) A_{i}\right)^{1 / 2} x_{0}\right\|^{2}+\left\|\left(A_{i}^{\prime} p_{i \Delta} S_{\Delta} A_{i}\right)^{1 / 2} x_{0}\right\|^{2}\right) .
$$


Hence, (A1) holds. For the general case, from the homogeneity property, one concludes that

$$
V^{n}\left(x_{0}, \theta_{0}\right)=E_{0}\left[\sum_{k=0}^{T_{1}-1}\left\|x_{k}\right\|^{2}+V^{n-1}\left(x_{T_{1}}, \theta_{T_{1}}\right)\right] .
$$

Since $V^{n-1}\left(x_{T_{1}}, \theta_{T_{1}}\right)=V^{n-1}\left(x_{\tau_{\Delta}}, \Delta\right) \mathbb{1}_{\left\{\tau_{\Delta} \leq T_{1}\right\}}+V^{n-1}\left(x_{T_{1}}, \theta_{T_{1}}\right) \mathbb{1}_{\left\{\tau_{\Delta}>T_{1}\right\}}$ then,

$$
V^{n}\left(x_{0}, \theta_{0}\right)=x_{0}^{\prime} S_{i}^{n} x_{0}=
$$

$$
E_{0}\left[\sum_{k=0}^{T_{1}-1}\left\|x_{k}\right\|^{2}+x_{T_{1}}^{\prime} S_{\Delta} x_{T_{1}} \mathbb{1}_{\left\{\tau_{\Delta} \leq T_{1}\right\}}+x_{T_{1}}^{\prime} S_{\theta_{T_{1}}}^{n-1} x_{T_{1}} \mathbb{1}_{\left\{\tau_{\Delta}>T_{1}\right\}}\right],
$$

which allow us to obtain

$$
\begin{gathered}
x_{0}^{\prime} S_{i}^{n} x_{0}=\sum_{k=0}^{\infty} p_{i i}^{k}\left(\left\|A_{i}^{k} x_{0}\right\|^{2}+\left\|\left({A_{i}^{\prime}}^{k+1} p_{i \Delta} S_{\Delta} A_{i}^{k+1}\right)^{1 / 2} x_{0}\right\|^{2}+\right. \\
\left.\left\|\left(A_{i}^{\prime k+1} \mathcal{E}_{i}^{\Delta}\left(\mathbf{S}^{\mathbf{n}-\mathbf{1}}\right) A_{i}{ }^{k+1}\right)^{1 / 2} x_{0}\right\|^{2}\right),
\end{gathered}
$$

and consequently $S_{i}^{n}-p_{i i} A_{i}^{\prime} S_{i}^{n} A_{i}=I_{n}+A_{i}^{\prime} p_{i \Delta} S_{\Delta} A_{i}+A_{i}^{\prime} \mathcal{E}_{i}^{\Delta}\left(\mathbf{S}^{\mathbf{n}-\mathbf{1}}\right) A_{i}$.

\section{References}

[1] E. Çinlar, "Introduction to Stochastic Processes", Prentice-Hall, 1975.

[2] O.L.V. Costa, M.D. Fragoso, R.P. Marques, "Discrete-Time Markov Jump Linear Systems", Probability and its Aplications, Springer, New York, 2005.

[3] O.L.V. Costa, M.D. Fragoso, Stability results for discrete-time linear systems with Markovian jumping parameters, Journal of Mathematical Analysis and Applications, 179 (1993), 154-178.

[4] J.B.R. do Val, C. Nespoli, Y.R.Z. Cáceres, Stochastic stability for Markovian jump linear systems associated with a finite number of jump times, Journal of Mathematical Analysis and Applications, 285, No. 3 (2003), 551-563.

[5] G.H. Golub, C.F. Van Loan, "Matrix Computation", John Hopkins Univ. Press, 1996.

[6] Y. Ji, H.J. Chizeck, K.A. Loparo, Stability and control of discrete-time jump linear systems, Control Theory and Advanced Technology, 7 (1991), 247-270.

[7] Y. Ji, H.J. Chizeck, Jump linear quadratic Gaussian control: Steady-state solution and testable conditions, Control Theory and Advanced Technology, 6, No. 3 (1990), 289-319.

[8] K. Zhou, J.C. Doyle, K. Glover, "Robust and Optimal Control", Prentice-Hall, 1996. 